UCID- 21077

\title{
Steady-State Approximations in Raman Excitation Equations
}

\author{
B.W. Shore
}

Theoretical Atomic and Molecular Physics Group

December 20, 1987

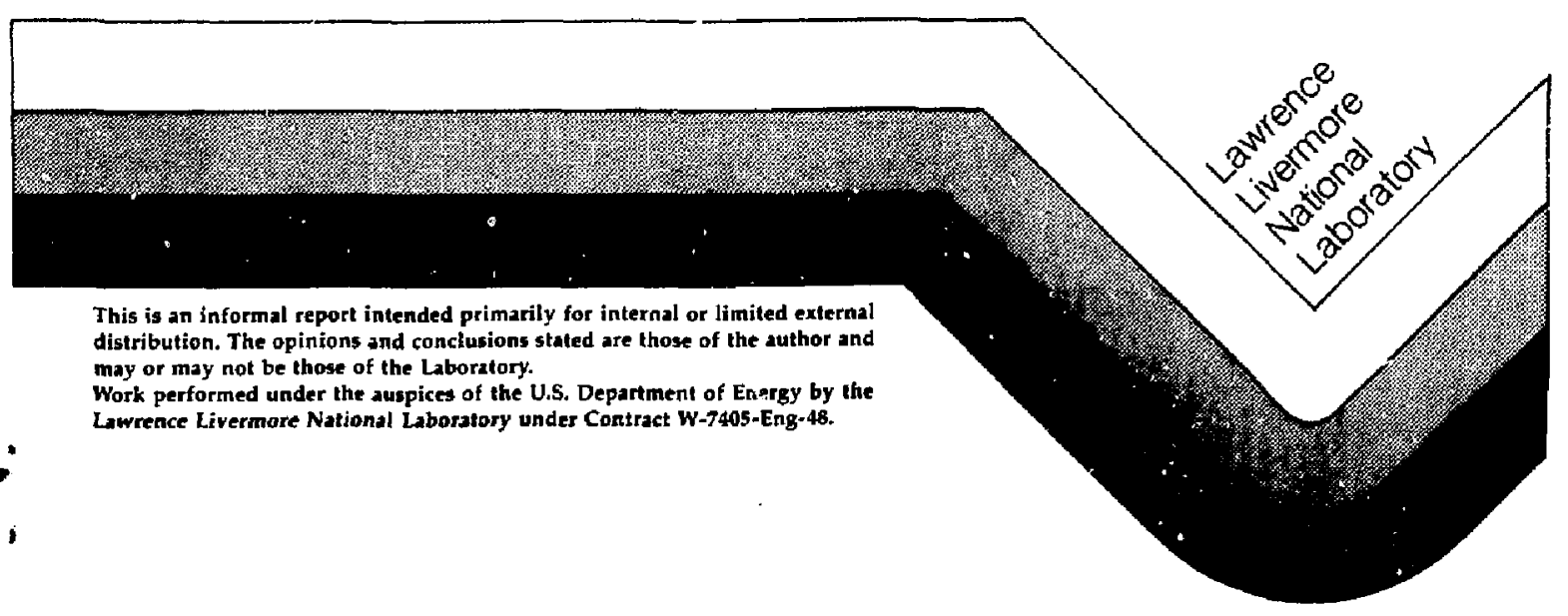


BWS 20 Dec 87

UCID- -21077

DE88 ن11759

\title{
Steady-State Approximstions in Raman Excitation Equations
}

\author{
B. W. Shore
}

Lawrence Livermore National Laborafory

\begin{abstract}
This memo presents equations describing propagation of beacrs of nearly mobochromatic radiation through a Raman-active molecular vapor, under conditions of steady excitation of quantum-mechanical molecules by classical radiation (that is, by radiation that comprises a carge number of photons per mode). Such conditions apnly in the continuous-wave experiments that are conducted to measure such properties as Raman gain or Raman scattering cross sections. Under those circumstances the propagation is described by rate equations for molecular excitation and rate equations for photon numbers.
\end{abstract}

Although these conditions do not apply for short-pulse transient excitation, both types of processes are parameterized by the same molecular polarizibility matrix elements. This memo discusses those parameters, as extracted from steady state observations.

This work was performed wider the auspices of the United States Department of Energy by the Lawrence Livermore National Laboratory under contract no. W-7405-Eng-18

\section{DISCLAIMER}

\begin{abstract}
This report was prepared as an account of work sponsored by an agency of the United States Government. Neither the United States Government nor any agency thereof, nor any of their employes, makes any warranty, express or implied, or assumes any legal liability or responsibility for the accuracy, completeness, or usefulness of any information, apparalus, product, or process disclowed, or represents that its use would not infringe privately owned rights. Reference herein to any specific commercial product, process, or service by trade name, trademark, manufacturer, or otherwise does not necessarily constitute or imply its endorsement, recommendation, or favering by the United Stales Gorrernment of any agency theroof. The views and opinions of authors expressed herein do not necessarily state or reflect thoce of the Unitod States Governmeat or any agency thereof.
\end{abstract}




\section{\$1. Introduction}

The propagation of radiation through a molecular medium is described by the wave equation

$$
\nabla^{2} \mathbf{E}-\frac{1}{c^{2}} \frac{\partial^{2}}{\partial t^{2}} \mathbf{E}=\frac{4 \pi}{c^{2}} \frac{\partial^{2}}{\partial t^{2}} \mathbf{P}
$$

where $\mathbf{E}$ is the solenoidal portion of the electric field and $\mathbf{P}$ is the solenoidal part of the polarization field. The theory of pulse propagation in the plase-wave approximation is based upon splitting the fields into positive and negative frequency components, as in $\mathbf{E}=\mathbf{E}^{(+)}+\mathbf{E}^{(-)}$, and the subsequent introduction of pulse envelope functions $\mathscr{E}_{\lambda}$ through the expansion

$$
E^{(+)}=\frac{J}{2} \sum_{\lambda} e(\lambda) f_{\lambda} \exp \left[i\left(k_{\lambda} \cdot r-j \omega_{\lambda} t\right]\right.
$$

with similar expansion for the polarization field $\mathrm{P}^{(+)}$in terms of envelope functions $\mathscr{P}_{\lambda}$. We wish to examine special cases of propagation in which the radiation is described by a set of collinear plane waves, and the polarization field originates in the steady weak-excitation response of molecules.

\section{The Basic Field Equations}

In the plane-wave slowly-varying envelope approximation the electric field and polarization field euvelope functions satisfy the first order equation

$$
\frac{\partial}{\partial z} \mathscr{G}_{\lambda}=\frac{2 \pi i}{G} \omega_{\lambda} \mathscr{P}_{\lambda}
$$

The mode envelope $\mathscr{B}_{\lambda}$ is characterized by a siowly varying phase and amplitude. The polarization mode appearing here is from the total polarization field (linear and nonlinear), so the dispersion relation is $c k_{\lambda}-\omega_{\lambda}$ and the mode intensity is

$$
I_{\lambda}=\frac{c}{8 \pi}\left|\mathscr{E}_{\lambda}\right|^{2}
$$

When we treat steady radiation, rather than pulses, we obcain a similar equation involving the nonlinear polarization and the refractive index $\eta_{\lambda}$,

$$
\frac{\partial}{\partial z} \mathscr{E}_{\lambda}=\frac{2 \pi i \omega_{\lambda}}{c \eta_{\lambda}} \operatorname{son} \mathscr{D}_{\lambda}
$$

The mode intensity is now 


$$
I_{\lambda}=\frac{c \eta_{\lambda}}{8 \pi}\left|E_{\lambda}\right|^{2}, \quad c k=\omega_{\lambda} \eta_{\lambda}
$$

The refractive index $\eta_{\lambda}$ appearing here is the ratio of $c k_{\lambda}$ to $\omega_{\lambda}$. It follows from these definitions that the mode intensity $I_{\lambda}$ satisfies the equation

$$
\left.\frac{\partial}{\partial z} I_{\lambda}=-\frac{1}{2} \omega_{\lambda} g_{m\left[\mathscr{E}_{\lambda}\right.}^{*} \mathscr{P}_{\lambda}\right] \text {. }
$$

These equations can be integrated once we have an algorithm for constructing the polarization mode envelope $\mathscr{P}_{\lambda}$.

\section{The Raman Polarization Mode}

For Raman processes in a vapor (randomly positioned molecules) of molecule density $\mathcal{N}$ [molecules per unit volume] the pulse polarization envelope needed as a source field for the electric-field envelope is prescribed as

$$
\begin{aligned}
& \mathscr{P}_{\lambda}(r, t)=\mathcal{N}(r, t) \sum_{p p^{\prime} \lambda^{\prime}} P_{p^{\prime} p}(r, t)\left\langle p\left|X\left(\omega_{\lambda^{\prime}}\right)\right| p^{\prime}\right): E\left(\lambda, \lambda^{\prime}\right) \mathcal{E}_{\lambda^{\prime}}(r, t) \\
& \quad x \exp \left[i\left(K_{p^{\prime}}-K_{p_{p}}+k_{\lambda^{\prime}}-k_{\lambda}\right) \cdot r\right] \exp \left[-i\left(W_{p^{\prime}}-W_{p^{\prime}}+\omega_{\lambda^{\prime}}-\omega_{\lambda}\right) t\right]
\end{aligned}
$$

where $\rho$ is the molecular density matrix, $\hbar W_{p}$ is the snergy of molecular state $p$, $\mathbf{X}(\omega)$ is the frequency-dependent molecular polarizibility operator, and $F\left(\lambda^{\prime}, \lambda\right)$ is a field polarization tensor formed from components of the unit vectors $e\left(\lambda^{\prime}\right)^{*}$ and $e(\lambda)$. The Cartesian components of this tensor are

$$
\epsilon_{\mathrm{j} j}\left(\lambda^{\prime}, \lambda\right)=e_{\mathrm{i}}\left(\lambda^{\prime}\right)^{*} e_{\mathrm{j}}(\lambda) \text {. }
$$

The notation $X: \in$ denotes the scalar formed by complete contraction of two second rank tensors, either by means of Cartesian components or by irreducible components,

$$
X: \epsilon=\sum_{i j} x_{i j} \epsilon_{i j}-\sum_{k q}(-1)^{q} X_{q}^{\left(k_{j}\right.} \epsilon_{-q}^{(k)} \text {, }
$$

In order that the envelope be slowly varying it is neccessary that the carrier frequencies of the electric field modes and those of the polarization field modes differ by the molecular excitation energy.

$$
\omega_{\lambda}-\omega_{\lambda^{\prime}} \simeq w_{p^{\prime}}-w_{p^{\prime}}
$$

By using the mode expansion for the poiarization field we can write the propagation equation for the electric field envelopes as a set of coupled equations 


$$
\frac{\partial}{\partial z} \mathscr{E}_{\lambda}=\sum_{\lambda \lambda^{\prime}} \mathbf{G}_{\lambda^{\prime}} \mathscr{E}_{\lambda^{\prime}}
$$

The gain matrix that is needed in this equation is obtainable from the expression

$$
\begin{aligned}
G_{\lambda^{\prime}}(r, t)= & \mathcal{N}(r, t) \frac{2 \pi i}{w_{\lambda}} \sum_{p p^{\prime} \lambda^{\prime}} \rho_{p^{\prime}}(r, t)\left(p \mid X\left(w_{\lambda^{\prime}}, X^{\prime}\right): E\left(\lambda_{,} \lambda^{\prime}\right)\right. \\
& \pi \exp \left[i\left(K_{p^{\prime}}-K_{p^{\prime}}+k_{\lambda^{\prime}}-k_{\lambda}\right) \cdot r\right] \exp \left[-i\left(W_{p^{\prime}}-W_{p^{\prime}}+w_{\lambda^{\prime}}-w_{\lambda}\right) t\right]
\end{aligned}
$$

The various carrier frequencies $\omega_{\lambda}$ and wavevectors $k_{\lambda}$, and the molecular parameters $W_{p}$ and $K_{p}$, are assumed to be chosen a priori. The molecular aumber density $\mathcal{N}$ is determined from initial physical conditions.

\section{The Molecular Equation}

Molecular properties enter the propagation equation through the density matrix $\rho_{\mathrm{pp}}$. Of particular importance are the off-diagonal elementr: (the coherences). These are responsible for induced dipole moments that produce Raman processes. These elements vanish in the absence of the radiation field, and so they must be determined in the presence of the radiation; it is these elements for which we require steady-state values.

The elements of the moiecular density matrix satisfy the equation

$$
\begin{aligned}
\frac{\partial}{\partial t} \rho_{p^{\prime} p} & =+\pi W_{p p^{\prime}}-W_{p^{\prime} p^{\prime}}+i \Gamma_{p^{\prime} p} \rho_{p^{\prime} p}-i W_{p^{\prime} p}\left[\rho_{p p}-\rho_{p^{\prime} p^{\prime}}\right] \\
& -i \sum_{p}\left[W_{p^{\prime} p^{\prime \prime}} \rho_{p^{\prime} p}-\rho_{p^{\prime} p^{\prime}} W_{p^{\prime} p}\right] .
\end{aligned}
$$

The matrix elments of the effective Hamiltonian that describes Raman processes are

$$
\begin{aligned}
& A W_{p^{\prime} p}(r, t)=-\frac{1}{4} \sum_{\lambda^{\prime}}\langle p| X\left(w_{\lambda} X p\right\rangle: \in\left(\lambda^{\prime}, \lambda\right) G_{\lambda^{\prime}} *(r, t) E_{\lambda}(r, t) \\
& \left.\quad x \exp \left[1 K_{p}-K_{p^{\prime}}+k_{\lambda}-k_{\lambda^{\prime}}\right), r\right] \exp \left[-r\left(W_{p}-W_{p^{\prime}}+w_{\lambda}-w_{\lambda^{\prime}}\right) t\right]
\end{aligned}
$$

The summation over modes is constrained by the requirement

$$
w_{p}+w_{\lambda} \simeq w_{p^{\prime}}+w_{\lambda^{\prime}}
$$


but the exponential exp $\left[-i\left(W_{p}-W_{p^{\prime}}+w_{\lambda}-w_{\lambda}\right) t\right]$ need not be unity if we are using temporal pulse mode expansions. (If we use temporal Fourier expansions, then we have a frequency matching condition that makes this exponential either unity or zero.).

This combination of equations (the coupled Maxwell-Bloch equations) applies to short pulses as well as to long pulses, and to transient phenomena as weil as steady state.

\section{Steady State Polarization}

Many experiments are conducted using $\mathrm{cw}$ radiation. Considerable literature is devoted to such cases. It is therefore useful to note how the present approach of pulse-mode expansions and density matrices produces results in that limit.

In steady state one often relates the Cartesian components of the polarization field to those of the eiectric field by a sequence of tensor susceptibilities, in the form

$$
P_{i}=\sum_{j} x_{i j}^{(2)} E_{j}+\sum_{j k} x_{j \mathrm{jk}}^{(\mathrm{g})} \mathrm{E}_{\mathrm{j}} \mathrm{E}_{\mathrm{k}}+\sum_{\mathrm{jk} \ell} x_{\mathrm{ijk \ell}}^{(1)} \mathrm{E}_{\mathrm{j}} \mathrm{E}_{\mathrm{k}} \mathrm{E}_{\ell}+\ldots
$$

The index of refraction $\eta$ has a similar expression,

$$
\eta=\eta_{0}+\eta_{2}|\mathbf{E}|^{2}+\eta_{1}|\mathbf{E}|^{*}+\ldots
$$

This memo discusses expressions for the nonlinear susceptibility tensors $x^{(3)}, x^{(4)}, \ldots$ and the nonlinear contributions to the index of refraction $\eta_{2}, \eta_{4}, \ldots$ derived on the basis of steady response of the molecules to the radiation field.

To derive the required equations we first describe the rate equation limit of the density matrix equation, as derived from the semiclassical Hamilionian (i.e. a description in which it is assumed that there are large numbers of photons present in any occupied mode, so that the fields may be treated classically).

We shall see below that this approximation produces propagation equations of the form

$$
\frac{\partial}{\partial z} \mathscr{E}_{\lambda}-i \mathscr{K}_{\lambda} \mathscr{E}_{\lambda}=\sum_{\lambda_{1}} G^{(1)}\left(\lambda_{j} \lambda_{1}\right) \mathscr{E}_{\lambda_{2}}+\sum_{\lambda_{1} \lambda_{2} \lambda_{3}} G^{(3)}\left(\lambda_{i} \lambda_{3} \lambda_{2}, \lambda_{1}\right) \mathscr{E}_{\lambda_{3}} \cdot \mathscr{E}_{\lambda_{2}} \mathscr{E}_{\lambda_{2}}
$$

where $G^{(t)}$ is, apart from a factor $2 \pi i / \omega$, a susceptibililty $x^{(t)}$ of order $n$. We shall see that this approximation also produces molecular-population rate equations

$$
\frac{\partial}{\partial t} \rho_{p_{p}^{\prime}}=\sum_{p} \rho_{p p} R_{p p^{\prime}}
$$


BWS 20 Dec 87

steady $1-6$

with rate coefficients of the form

$$
R_{\mathrm{pp}}=\frac{1}{8 \hbar^{2}} g_{m} \sum_{\lambda_{1} \lambda_{2} \lambda_{2} \lambda_{4}} x^{(j)}\left(\lambda_{1} \lambda_{3} ; \lambda_{2} \lambda_{1}\right) \mathscr{E}_{\lambda_{4}} * \mathscr{E}_{\lambda_{3}} * \mathscr{E}_{\lambda_{2}} \mathscr{E}_{\lambda_{1}}
$$

$(\mathrm{V} .1-21)$

These rate coefficients can also be derived by time-dependent porturbation theory (the Golden Ruile rates) for radiation described by photon-number states. Such states are useful for describing spontaneous emission in a fully quantum mechanical way. 


\section{The Steady-State Density Matrix}

Consider quasi steady-state excitation, in which the off-diagonal elements of $\rho(t)$ adiabatically follow the diagonal elements. Following the traditional method (e.g. Wilcox and Lamb) we set to zero the time derivatives of the off-diagonal elements, and obtain the equations

$$
\begin{gathered}
0=-i W_{p^{\prime} p}\left[\rho_{p p}-\rho_{p^{\prime} p^{\prime}}\right]+i \rho_{p_{p}^{\prime}}\left[W_{p_{p}}-W_{p^{\prime} p^{\prime}}+i \Gamma_{p^{\prime} p}\right] \\
-i \sum_{p^{\prime \prime} \neq p, p^{\prime}}\left[W_{p^{\prime} p^{\prime \prime}} \rho_{p^{\prime} p}-\rho_{p^{\prime} p^{\prime}} W_{p^{\prime \prime} p}\right]
\end{gathered}
$$

These form a set of linear equations to be solved for the off-diagonal elements. In general the solution requires inversion of a matrix. This set of equations simplifies if we neglect all but one off-diagonal element, say $\rho_{p}(t)$. This approximation is appropriate for weak excitation, in which no population transfer occurs. Let us introdece the notation

$$
\Delta_{p}=W_{p p}
$$

for the diagonal elements of the matrix $W_{p p}(t)$ in the steady-state limit. This represents a steady-state dynamic Stark shift of state $p$. Then we obtain the result

$$
\rho_{p^{\prime} p}=\frac{\left[\rho_{p p}-\rho_{\left.p^{\prime} p^{\prime}\right]}\right.}{\left[\Delta_{p}-\Delta_{p^{\prime}}+\bar{T}_{p^{\prime} p}\right]} W_{p^{\prime} p^{\prime}}
$$

The Hamiltonian matrix elements needed for the present applieation are those of the Raman Hamiltonian, Eqn. (V.1-6). Substitution gives the expression

$$
\begin{gathered}
\rho_{p^{\prime} p}(r, t)=-\frac{1}{4 \hbar} \frac{\left[\rho_{p p}(r, t)-\rho_{p^{\prime}}(r, t)\right]}{\left[\Delta_{p}-\Delta_{p^{\prime}}+i \Gamma_{p_{p}^{\prime}}\right]} \sum_{\lambda \lambda^{\prime}}\left\{p \nmid X\left(\omega_{\lambda} X_{p}\right): \in\left(\lambda^{\prime}, \lambda\right) \mathscr{E}_{\lambda^{\prime}}(r, t)^{*} \mathscr{E}_{\lambda}(r, t)\right. \\
\quad x \exp \left[i\left(K_{p}-K_{p^{\prime}}+k_{\lambda}-k_{\lambda^{\prime}}\right) \cdot r\right] \exp \left[-j\left(W_{p}-W_{p^{\prime}}+w_{\lambda}-w_{\lambda^{\prime}}\right) t\right] .
\end{gathered}
$$

This formula expresses the off-diagonal elements of the density matrix (the coherences) in terms of the diagonal elements (the populations). The summation over modes is restricted by the RWA condition that

$$
w_{p}-w_{p^{\prime}}=w_{\lambda^{\prime}}-w_{\lambda}
$$

It is possible to proceed to further approximations in which we take the populations to be those of thermodynamic equilibrium, which is the case prior to exposure to the pulsed radiation. However, it is not neccessary to make this further approximation. One can derive population rate equations, as noted below, which take into account the radiation field in establishing the steady state. 


\section{Steady-State Polarization}

We now substitute this approximation for the density matrix into the expression (V.1-4) for the polarization field. Upon replacing the elements of the density matrix by their steady-state limit, we obtain the result

$$
\begin{aligned}
& \mathscr{P}_{\lambda}(r, t)=\mathcal{N}(r, t) \sum_{p \lambda_{1}} \rho_{p p}\left(p\left|X\left(\omega_{1}\right)\right| p\right): E\left(\lambda, \lambda_{1}\right) \mathcal{E}_{\lambda_{2}}(r, t) \exp \left[-i\left(\omega_{2}-\omega_{\lambda}\right) t\right] \\
& +\frac{\mathcal{N}(r, t)}{4 \hbar} \sum_{p p^{\prime} \lambda_{1} \lambda_{2} \lambda_{7}} \frac{\left[\rho_{p p}-\rho_{p^{\prime} p^{\prime}}\right]}{\left[\Delta_{p^{\prime}}-\Delta_{p}+T_{p p^{\prime}}\right]} \mathcal{E}_{\lambda_{2}}(r, t) \mathcal{E}_{\lambda_{2}}(r, t)^{*} \mathscr{E}_{\lambda_{1}}(r, t) \\
& x \exp \left[i\left(k_{1}-k_{2}+k_{3}-k_{\lambda}\right) \cdot r\right] \exp \left[-j\left(\omega_{1}-\omega_{2}+\omega_{3}-\omega_{\lambda}\right) t\right] \delta\left(W_{p} \cdot-W_{p}+\omega_{1}-w_{2}\right) \\
& \left.x\left(p\left|X\left(\omega_{1}\right)\right| p^{\prime}\right): \in\left(\lambda_{2}, \lambda_{1}\right) \quad(p\rceil X\left(\omega_{1}\right) \lambda p\right): \in\left(\lambda, \lambda_{3}\right) \text {. }
\end{aligned}
$$

This is the perturbation-theory approximation to the polarization field. We sum these terms over all contributions whose exponentiated frequencies lie within the assigned bandwidth. There remains a slow time dependence of this amplitude from the time dependence of the populations, as expressed by the diagonal matrix elements $\rho_{\mathrm{pp}}(\mathfrak{t})$. The formula has the following interpretation.

The first summation, involving a single electric field amplitude, produces contributions to $\mathscr{P}_{\lambda}$ only from those modes that have the same frequency. This is an elastic seattering contribution (Rayleigh scattering). This part is the linear part of the polarization field. However, this term also describes a polarization field whose polarization differs from that of the incident field.

The second summation exhibits those contributions to the polarization that accompany Raman transitions, i.e. the summation admits only terms for which $W_{p} \neq$ $W_{p}$. This sum is the nonlinear part of the polarization field. The factor $\delta\left(W_{p}-W_{p^{\prime}}+w_{1}-w_{2}\right)$ expresses the RWA condition on the steady-state density matrix The frequency condition on this part is

$$
\left|\omega_{2}-\omega_{1}-\omega_{3}+\omega_{4}\right|<\omega_{4}
$$

That is, the combination of three frequencies $\omega_{1}+\omega_{3}-\omega_{2}$ must fall within the bandwidth of the frequency for mode $\lambda_{4}$. Because we have assumed that the various field modes are all well separated, this condition en only be satisfied by a single frequency. (If the occuring frequency is not in our original catalos, then we must add it. If the sum is "not quite" zero, then we must redefine our pulse modes to include a broader range of frequencies.) We conclude that with temporal pube shapes, is with Fourier decomposition, the condition

$$
\omega_{2}-\omega_{1}-\omega_{3}+\omega_{4}=0
$$

applies. This expresses the conditions for Raman scattering and four-wave mixing. 
The summation is actually more restricted than condition (V.1-23) would indicate, because the factor $\rho_{\rho}^{\prime}$ contains a constraint that the difference of two frequencies be equal to a nonzero Raman transition frequency. This condition is

$$
\omega_{1}-\omega_{3}=-\left(\omega_{3}-\omega_{3}\right) \neq 0
$$

That is, we omit the elastic scattering case, for which the frequency condition is satisfied because $\omega_{1}=\omega_{2}$.

\section{Nonlinear Susceptibilities}

In steady state one of ten relates the Cartesian components of the polarization fieid to those of the electric field by a sequence of tensor susceptibilities, in the form

$$
P_{i}=\sum_{j} x_{i j}^{(1)} E_{j}+\sum_{j k} x_{j j k}^{(2)} E_{j} E_{k}+\sum_{j k \ell} \chi_{i j k \ell}^{(j)} E_{j} E_{k} E_{\ell}+\ldots
$$

We are particularly concerned here with $x^{(1)}$ and $x^{(3)}$. From Eqn. (V.2-6) we obtain an expression for the linear susceptibility tensor,

$$
x^{(1)}\left(\lambda ; \lambda_{1}\right)=\mathcal{N} \sum_{p \lambda_{1}} \rho_{p p}\left(p\left|X\left(\omega_{1}\right)\right| p\right): E\left(\lambda_{1} \lambda_{1}\right) \exp \left[-i\left(\omega_{1}-\omega_{2}\right) t\right]
$$

and for the third-order nonlinear susceptibility,

$$
\begin{aligned}
& x^{(s)}\left(\lambda_{i} \lambda_{3}, \lambda_{2}, \lambda_{1}\right)=\frac{M(r, t)}{4 \hbar} \sum_{p p^{\prime} \lambda_{1} \lambda_{1} \lambda_{3}} \frac{\left[p_{p p}-\theta_{\left.p^{\prime} p^{\prime}\right]}\right.}{\left[\Delta_{p^{\prime}}-\Delta_{p}+\pi \Gamma_{p p^{\prime}}\right]} \mathcal{E}_{\lambda_{3}}(r, t) \mathscr{E}_{\lambda_{2}}(r, t)^{*} \\
& \mathcal{G}_{\lambda_{1}}(r, t)
\end{aligned}
$$

$$
\begin{aligned}
& x \exp \left[i\left(k_{1}-k_{2}+k_{3}-k_{\lambda}\right) \cdot x\right] \exp \left[-j\left(\omega_{1}-\omega_{2}+\omega_{3}-\omega_{\lambda}\right) t\right] d\left(W_{p^{\prime}}-W_{p}+\omega_{1}-\omega_{2}\right) \\
& \times \quad\left(p \mid X\left(\omega_{1}\right) X p^{\prime}\right): \in\left(\lambda_{2}, \lambda_{1}\right) \quad\left\langle p\left|X\left(\omega_{3}\right)\right| p\right): \in\left(\lambda_{1} \lambda_{3}\right) .
\end{aligned}
$$

There are several conventions for defining nonlinear susceptibilities. Typically one defines the third-order fourth-rank Raman-procesy susceptibility tensor by the following relationship between Cartesian components:

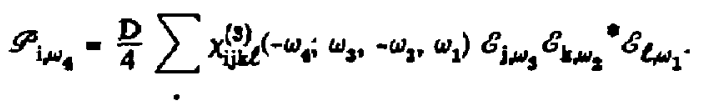

With this definition the frequency arguments of $x^{(3)}$ sum to zero (so that in the present example $\left.\omega_{1}-\omega_{3}-\omega_{2}+\omega_{1}\right)$. The factor $D$ expresses the number of distinct terms that contribute: 


$$
\begin{aligned}
& D=6 \text { if } \omega_{1}, \omega_{2} \text { and } \omega_{3} \text { are all distinct } \\
& D=3 \text { if two of the frequencies } \omega_{1}, \omega_{2}, \omega_{3} \text { are identical } \\
& D=1 \text { if } \omega_{1}=\omega_{2}=\omega_{3}
\end{aligned}
$$

For example, in the case of Stokes generation at frequency $\omega_{3}$ from laser frequency $\omega_{L}$ one has

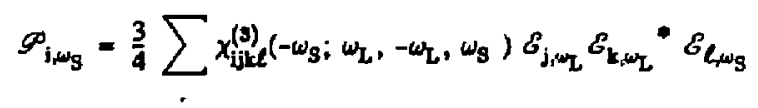

For anti-Stokes generation at frequency $\omega_{A}$ from the laser frequency $\omega_{L}$ one has

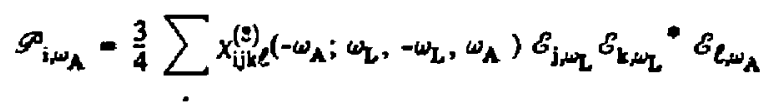

For the coupling between Stokes and Anti-Stokes frequencies one has



One may generalize the Cartesian representation to the form

$$
\mathscr{S}_{\lambda}=\frac{\mathrm{D}}{4} \sum x^{(3)}\left(\lambda_{1} \lambda_{2}, \lambda_{2}, \lambda_{1}\right) \mathscr{E}_{\lambda} \mathscr{E}_{\lambda_{2}} \cdot \mathscr{E}_{\lambda_{1}}
$$

\section{Strong Pump Mode}

As an example of the application of this last formuls, suppose that the pump mode is the strongest field, and other modes are much weaker. We seek a solution for the Stokes wave polarization field, $\omega_{4}=\omega_{3}$. The three possibilities that satisfy the frequency requirement $\omega_{1}+\omega_{2}-\omega_{2}=\omega_{4}$ are

$$
\begin{array}{ll}
\omega_{3}=\omega_{3}, & \omega_{2}=\omega_{1}=\omega_{3}, \\
\omega_{3}=\omega_{3}, & \omega_{2}=\omega_{1}=\omega_{p}, \\
\omega_{1}=\omega_{3}, & \omega_{2}=\omega_{3}=\omega_{P},
\end{array}
$$

The first possibility involves only Stokes fields, $s 0$ by our hyrothesis of strong fump, it is less importint than processes involving two pump amplitudes. The second choice describes elastic scattering. Only the final choice occurs in Raman transitions. Thus, allowing for the possibility that the Stokes polarization field may differ in polarization from the existing Stokes field, we write 


$$
\begin{aligned}
& g_{\mathrm{g}}(t)=\frac{\mathcal{N}}{4 \hbar} \sum_{p p^{\prime} S^{\prime}} \frac{\left[\rho_{p p}-\rho_{\left.p^{\prime} p^{\prime}\right]}\right.}{\Delta_{p}-\Delta_{p^{\prime}}+i \Gamma_{p^{\prime}}} \mathscr{S}_{p^{\prime}}(t) \mathscr{E}_{p}(t)^{*} \mathscr{S}_{\mathrm{S}^{\prime}}(t) \\
& x \exp \left[i\left(k_{\mathbf{g}^{\prime}}-k S\right) . r\right] \&\left(W_{p^{\prime}}-W_{p}+w_{g^{\prime}}-w_{p}\right) \\
& \left.x\left(p \mid X\left(\omega_{p}\right) p\right): \in(S, P)\langle p| X\left(\omega_{g^{+}}\right) \backslash p\right\}: \in\left(P, S^{\prime}\right)
\end{aligned}
$$

This expression must be summed over magnetic sublevels or molecular orientation. From this expression we deduce the gain matrix element

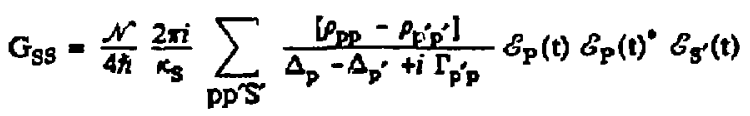

$$
\begin{aligned}
& x \exp \left[\left(i\left(k_{g^{\prime}}-k S\right), r\right] S\left(W_{p^{\prime}}-W_{p}+w_{g^{\prime}}-w_{p}\right)\right. \\
& \left.x\left\{p\left|X\left(\omega_{p}\right)\right| p\right): E(S, P)\left\{p \mid X\left(\omega_{\mathcal{B}^{\prime}}\right)\right\} p\right\}: E\left(P, S^{\prime}\right)
\end{aligned}
$$

By comparing Eqn. (V2-17) with the expression

$$
\mathscr{S}_{\mathrm{S}}=\frac{3}{4} x_{\mathrm{SPP}}^{(9)}\left(-\omega_{\mathrm{S}} ; \omega_{\mathrm{P}},-\omega_{\mathrm{P}}, \omega_{\mathrm{S}}\right) \mathscr{E}_{\mathrm{P}} \mathcal{E}_{\mathrm{P}} \cdot \mathscr{E}_{\mathrm{S}^{\prime}}
$$

we obtain the following expression for the susceptibility.

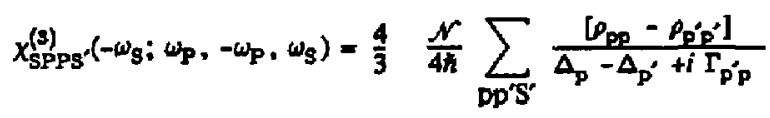

$$
\begin{aligned}
& x \exp \left[i\left(k_{g}-k_{g}\right) \cdot r\right] \delta\left(w_{p^{\prime}}-w_{p}+w_{g^{\prime}}-w_{p}\right) \\
& x\left(p 1 X\left(\omega_{P}\right) \mid p\right): \in(S, P)\left(p \mid X\left(\omega_{S^{\prime}}\right) \backslash p^{\prime}\right): E\left(P, S^{\prime}\right)
\end{aligned}
$$




\section{\$3. The Steady-State Amplitude Propagation Equation}

Let us substitute this expression for the polarization field into the pulse propagation equation. In so doing we wish to separate out those terms that involve the particular electric-field envelope whose propagation depends upon the polarization envelope. The envelope of interest may occur either as the single term of Rayleigh scattering or it may occur as one of the triad of envelopes that constitute Raman processes. The sums over modes are constrained in frequencies, but they must still involys all possible polarizations. To emphasize this, let us here replace the mode index $\lambda$ by a frequency label $\omega$ and a polarization label $\mu$. We can then write the propagation equation for mode $\lambda=\mu, \omega$ as

$$
\begin{aligned}
& \frac{\partial}{\partial z} \mathscr{E}_{\mu \omega}-i \mathscr{K}_{\mu \omega} \mathscr{E}_{\mu \omega}=\mathscr{N} \frac{2 \pi i}{c} \omega \sum_{p} \rho_{p p} \sum_{\mu_{i} \neq \mu} \mathscr{E}_{1}(t)(p ; \mathbf{X}(\omega) \mid p): \in\left(\mu, \mu_{2}\right) \\
& +\frac{\mathscr{N}_{\pi}}{2 \hbar \mathrm{c}} \omega \sum_{p p^{\prime}} \frac{\left[\rho_{\mathrm{pp}}-\rho_{\left.\mathrm{p}^{\prime} \mathrm{p}^{\prime}\right]}\right.}{\left[\Delta_{\mathrm{p}}-\Delta_{\mathrm{p}^{\prime}}+\pi_{\mathrm{p}^{\prime} \mathrm{p}}\right]} \mathscr{E}_{3}(t) \mathscr{E}_{2}(t)^{*} \mathscr{E}_{1}(t) \\
& x \exp \left[i\left(k_{1}-k_{2}+k_{3}-k\right) x\right] \exp \left[-i\left(\omega_{1}-\omega_{2}+\omega_{3}-\omega\right) t\right] \delta\left(W_{p^{\prime}}-W_{p}+\omega_{3}-\omega_{2}\right) \\
& \left.x\{p\} \mathbf{X}\left(\omega_{1}\right) \backslash p\right\rangle: \in\left(\mu_{2}, \mu_{1}\right)\left\langle p\left|X\left(\omega_{3}\right)\right| p^{\prime}\right): \in\left(\mu, \mu_{3}\right) \text {. }
\end{aligned}
$$

in which the second sum is constrained by the frequency matching requirement but excludes the cases $\lambda_{1}=\mu w$ and $\lambda_{3}=\mu w$. The coefficient $\mathscr{K}_{m}$ is the effective wavenumber of the pulse mode, given by

$$
\begin{aligned}
& \mathscr{R}_{\mu \nu}=\frac{\omega}{\varepsilon} 2 \pi \mathscr{N} \sum_{\mathrm{p}} \rho_{\mathrm{pp}}(\mathrm{p}|\mathbf{X}(\omega)| \mathrm{p}): E(\mu, \mu) \\
& +\frac{\omega}{c} \frac{\mathcal{N}_{\pi}}{2 \hbar} \sum_{p p^{\prime}}\left[\rho_{p p}-p_{p^{\prime} p^{\prime}}\right]\left[\frac{\left[\Delta_{p}-\Delta_{p^{\prime}}-i \Gamma_{p_{p}^{\prime}}\right]}{\left[\left(\Delta_{p}-\Delta_{p^{\prime}}\right)^{2}+\left(\Gamma_{p_{p}^{\prime}}\right)^{2}\right]}\right] \\
& x \sum_{\lambda_{1} \lambda_{2}} \mathscr{E}_{2}(t)^{*} \mathscr{E}_{1}(t) \delta\left(w_{1}-w_{2}\right) \delta\left(k_{1}-k_{2}\right) \delta\left(W_{p^{\prime}}-W_{P}+w_{1}-w_{2}\right) \\
& x\left[\left(p|X(\omega)| p^{\prime}\right\rangle: \in\left(\lambda_{2} \lambda_{1}\right)\langle p| X\left(\omega_{1}\right) p\right): \in\left(\lambda_{2}, \lambda\right) \\
& \left.\left.+\left\langle p^{\mid}|X(\omega)| p^{\prime}\right): \in\left(\lambda_{2}, \lambda\right)\langle p| X\left(\omega_{1}\right) \backslash p\right): \in\left(\lambda_{2}, \lambda_{1}\right)\right]
\end{aligned}
$$

Apart from the factor $\omega / c$, this is the complex index of refraction for frequency $\omega$ 
and polarization $\mu$ :

$$
F_{\omega \omega}=\frac{\omega}{c} \eta(\mu, \omega)
$$

Note that in the RWA the second sum is restricted to cases for which $W_{p}=W_{p}$.

\section{The Nonlinear Index}

A portion of this expression for $K_{\lambda}$ may be regarded as the $\eta_{2}(\lambda)$ contribution to the nonlinear index of refraction. That portion is, by definition, proportional to the square of the electric field (or intensity) and so it involves the irreducible component $E(\lambda, \lambda)^{0}$ of the field tensor.

$$
\begin{aligned}
& I_{\lambda}=\frac{c \eta_{\lambda}}{8 \pi}\left|E_{\lambda}\right|^{2}=\frac{c \eta_{\lambda}}{2 \pi} E_{\lambda}(-) \cdot E_{\lambda}(+)=\frac{c \eta_{\lambda}}{8 \pi}\left|E_{\lambda}\right|^{2} \sum_{i} E_{i j}(\lambda, \lambda) \\
& \sum_{i} E_{i j}(\lambda, \lambda)=-\sqrt{3} \in\left(\lambda^{\prime}, \lambda\right)^{0}-\&\left(\lambda, \lambda^{\prime}\right)
\end{aligned}
$$

In situations where only one polarization is present it is possible to define a nonlipear index of refraction by the expression

$$
c \mathscr{R}_{\omega \omega}=\omega \eta(\omega)=\omega\left[\eta_{0}(\omega)+\eta_{2}(\omega) \sum_{\omega^{\prime}} \pm\left|E_{\omega \omega}\right|^{2}\right]
$$

Under these restrictions the nonlinear index $\eta_{2}(\lambda)$ is

$$
\begin{aligned}
\eta_{2}(\omega, \mu) & =2 \frac{\mathcal{N} \pi}{\hbar} \sum_{p p^{\prime}}\left[\rho_{p p}-\rho_{p^{\prime} p^{\prime}}\right] \frac{\left[\Delta_{p}-\Delta_{p^{\prime}}-i \Gamma_{p_{p}^{\prime}}\right]}{\left[\left(\Delta_{p}-\Delta_{p^{\prime}}\right)^{2}+\left(\Gamma_{p_{p}^{\prime}}\right)^{2}\right]} \\
& \left.\times 2 \operatorname{The}_{e}\left[\left(p\left|X(\omega)^{(0)}\right| p^{\prime}\right)(p) X\left(\omega_{1}\right)^{(0)} \mid p\right)\right]
\end{aligned}
$$

When several polarizations are present it is not possible to write the relationship between $\mathscr{K}_{\boldsymbol{m}}$ and $\omega$ in such a simple form.

\section{Propagation and Suscoptibility}

As can be seen from these results, the general form for the propagation equation, in the steady-state approximation to the density matrix, is 


$$
\begin{aligned}
\frac{\partial}{\partial z} E_{\lambda}-i \mathscr{E}_{\lambda} \mathscr{E}_{\lambda}=\left[\frac{2 \pi i \omega_{\lambda}}{\operatorname{cr} \gamma_{\lambda}}\right] \sum_{\lambda_{1}} x^{(1)}\left(\lambda ; \lambda_{1}\right) E_{\lambda_{1}} \\
+\left[\frac{2 \pi i \omega_{\lambda}}{\operatorname{cr} \eta_{\lambda}}\right] \sum_{\lambda_{1} \lambda_{2} \lambda_{3}} x^{(3)}\left(\lambda ; \lambda_{3}, \lambda_{2}, \lambda_{1}\right) E_{\lambda_{g}}^{*} E_{\lambda_{2}} E_{\lambda_{1}}
\end{aligned}
$$

The coefficients $x^{(k)}$ that appear here are susceptibilities. They are, from the preceding expressions, the first order susceptibility

$$
\begin{gathered}
\chi^{(1)}\left(\mu \omega ; \mu_{1}, \omega_{1}\right)=\delta\left(\omega-\omega_{1}\right) \exp \left[i\left(k_{2}-k_{2}+k_{1}-k\right) . r\right) \\
\times \sim \mathcal{P} \sum_{p} \rho_{p p}(p|X(\omega)| p): \in\left(\mu_{1} \mu_{1}\right)
\end{gathered}
$$

and the third order susceptibility

$$
\begin{aligned}
& \chi^{(3)}\left(\mu \omega, \mu_{3} \omega_{3}, \mu_{2} \omega_{2}, \mu_{1} \omega_{1}\right)=\delta\left(\omega+\omega_{3}-\omega_{1}-\omega_{2}\right) \exp \left[i\left(k_{2}-k_{3}+k_{1}-k\right) . r\right) \\
& x \frac{\mathcal{N}}{4 \hbar} \sum_{p p^{\prime}}\left[\rho_{p p}-\rho_{p^{\prime} p^{\prime}}\right] \frac{\left[\Delta_{p}-\Delta_{p^{\prime}}-t \Gamma_{p_{p}^{\prime}}\right]}{\left[\left(\Delta_{p}-\Delta_{p^{\prime}}\right)^{2}+\left(\Gamma_{p_{p}^{\prime}}\right)^{2}\right]}
\end{aligned}
$$

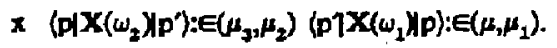

The Stimulated Raman Gain Coefficient

Let us consider the equation for the intensity propagation of a Stokes pulse in the presence of a strong pump pulse. The equation for Stokes intensity is

$$
\frac{\partial}{\partial z} I_{s}=-\frac{1}{2} \omega_{s} g_{m}\left[E_{g} * \mathscr{P}_{s}\right]
$$

We substitute into this equation the preceding expression for Stokes polarization, and obtain the result

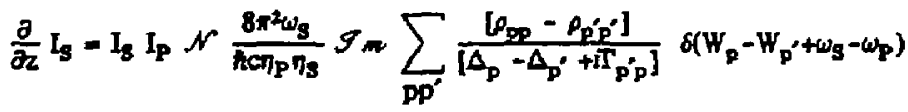

$$
\begin{aligned}
& x\left\langle p \mid X\left(\omega_{p}\right) \backslash p\right\rangle: E(S, P)\left(p \nmid X\left(\omega_{p}\right) \mid p\right): \in(S, P)
\end{aligned}
$$

Typically we apply this formula to cases in which the differences in dynamic Stark shifts $\Delta_{p}$ are negligible. Under those circumstances the equation becomes 


$$
\begin{aligned}
& \frac{\partial}{\partial z} I_{S}=I_{S} I_{P} \frac{8 \pi^{2} \omega_{g}}{\hbar c \eta_{P} \eta_{S}} \sum_{p p^{\prime}} \frac{\left[\mathcal{N}_{p^{\prime}}-\mathcal{N}_{p^{\prime}}\right]}{T_{p^{\prime} p}} \delta\left(W_{p^{\prime}}-W_{p^{\prime}}+\omega_{S}-\omega_{P}\right) \\
& x\left\langle p^{\prime}\left|X\left(\omega_{p}\right)\right| p\right): \in(S, P)\left\langle\left(p \mid X\left(\omega_{S}\right) X p^{\prime}\right): \in(P, S)\right.
\end{aligned}
$$

Here $\mathscr{N}_{\mathrm{p}} \neq \mathcal{N}_{\mathrm{pp}}$ is the number density of molecules in state $\mathrm{p}$.

The stimulated Raman gain coefficient $B_{S P}$ is defined by the equation

$$
\frac{d}{d z} I_{S}=8_{S P} I_{S} I_{P}+*_{S}
$$

where es represents the spontaneous emission source term for the Stokes radiation. This latter term has not been included in our derivation of the incensity propagation equation. We see that the stimulated gain coefficient can be written as

$$
\begin{aligned}
& g_{S P}=\frac{8 \pi^{2} \omega_{S}}{c \eta_{P} \eta_{S}} \sum_{D p^{\prime}} \frac{\left[\mathscr{N}_{p^{\prime}}-\mathscr{N}_{p^{\prime}}\right]}{\hbar \Gamma_{p^{\prime}}} \delta\left(W_{p^{\prime}}-W_{P^{\prime}}+\omega_{S}-\omega_{P}\right) \\
& \left.x\left(p^{\prime}\left|X\left(\omega_{p}\right)\right| p\right): \in(S, P)\langle p| X\left(w_{S}\right) \backslash p^{\prime}\right): \in(P, S)
\end{aligned}
$$

Here $p$ denotes the lower-lying level of the pair p,p. This expression must be summed over magnetic sublevels of the molecule. The gain coefficient can be expressed in terms of the third-order susceptibility as

$$
\mathrm{s}_{\mathrm{SP}}=\frac{32 \pi^{2}}{\eta_{\mathrm{P}}} \frac{\omega_{\mathrm{S}}}{\mathrm{c} \eta_{\mathrm{S}}} x_{\mathrm{SPPS}}^{(3)}\left(-\omega_{\mathrm{g}} ; \omega_{\mathrm{P}},-\omega_{\mathrm{P}}, \omega_{\mathrm{S}}\right)
$$




\section{The Rate Equation}

Traditional broadband excitation follows the description of rate equations. Let us note how those equations emerge from the present approach. We can use the approximation for off-diagonal elements of the density matrix in the equation for the diagonal elements, to obtain an equation that involves only diagonal elements (i.e. populations). The result is the rate equation

$$
\frac{d}{d t} \rho_{p_{p}^{\prime}}(t)=-\Gamma_{p^{\prime} p^{\prime}} \rho_{p^{\prime} p^{\prime}}(t)+2 g_{m} \sum_{p \neq p^{\prime}} W_{p p^{\prime}} \rho_{p^{\prime} p}
$$

For the Raman Hamiltonian this equation becomes

$$
\begin{aligned}
& \frac{d}{d t} \rho_{p^{\prime} p^{\prime}}(t)=-\Gamma_{p^{\prime} p^{\prime}} \rho_{p^{\prime} p^{\prime}}(t)+\frac{1}{2 \hbar} g_{m} \sum_{p \neq p^{\prime}} w_{p p^{\prime}} \frac{\left[\rho_{p p^{\prime}}-\rho_{\left.p^{\prime} p^{\prime}\right]}\right.}{\left[\Delta_{p}-\Delta_{p^{\prime}}+\pi \Gamma_{p^{\prime} p}\right]} \\
& x \sum_{\lambda \lambda^{\prime}} E_{\lambda^{\prime}}(t)^{*} \mathscr{E}_{\lambda}(t) \exp \left[-i\left(W_{p}-W_{p^{\prime}}+w_{\lambda}-w_{\lambda^{\prime}}\right) t\right]\left\langle p^{\prime}\right] X\left(\omega_{\lambda} X p\right): E\left(\lambda^{\prime}, \lambda\right)
\end{aligned}
$$

Upon substituting the formula for $W_{p^{\prime} p}$ we find the equation

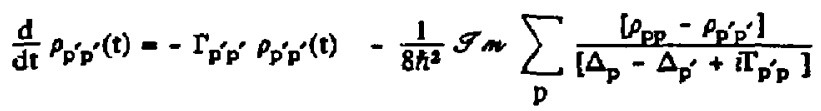

$$
\begin{aligned}
& x \sum_{\lambda_{1} \lambda_{2} \lambda_{2} \lambda_{1}} \mathscr{E}_{\lambda_{1}}(t)^{*} \mathscr{E}_{\lambda_{3}}(t) \mathscr{E}_{\lambda_{2}}(t)^{*} \mathscr{E}_{\lambda_{1}}(t) \exp \left[-i\left(\omega_{1}-\omega_{2}+\omega_{3}-\omega_{1}\right) t\right] \\
& x \quad\left(p\left|X\left(\omega_{3}\right)\right| p^{\prime}\right): \in\left(\lambda_{2}, \lambda_{3}\right)\left(p^{\prime}\left|X\left(\omega_{2}\right)\right| p\right): \in\left(\lambda_{2}, \lambda_{2}\right) .
\end{aligned}
$$

This equation has the appearance of a set of rate equations,

$$
\frac{d}{d t} \rho_{p^{\prime} p^{\prime}}=-\rho_{p^{\prime} p^{\prime}} R_{p^{\prime} p^{\prime}}+\sum_{p \rightarrow p^{\prime}} \rho_{p p} R_{p p^{\prime}}
$$

With the neglect of Stark shifts in the denominator, the rate coefficient is 
AWS 20 Dec 87

steady $1-17$

$$
\begin{aligned}
& R_{p p^{\prime}}=\frac{1}{8 h^{2}} \frac{1}{\Gamma_{p p^{\prime}}} \mathscr{E}_{*} \sum_{\lambda_{1} \lambda_{2} \lambda_{3} \lambda_{4}} E_{\lambda_{4}}(t)^{*} E_{\lambda_{3}}(t) \mathscr{E}_{\lambda_{2}}(t)^{*} E_{\lambda_{1}}(t) \\
& x \exp \left[-i\left(\omega_{2}-\omega_{2}+\omega_{3}-\omega_{4}\right) t\right]\left(p \mid X\left(\omega_{3}\right)^{\prime} p^{\prime}\right): E\left(\lambda_{4}, \lambda_{3}\right)\left\langle p^{\prime}\left|X\left(\omega_{1}\right)\right| p\right\rangle: \in\left(\lambda_{2}, \lambda_{2}\right) .
\end{aligned}
$$

(V.4-5)

The rate coefficients can also be written in terms of the nonlinear susceptibility intraduced earlier.

$$
R_{p p^{\prime}}=\frac{1}{8 \hbar^{2}} g_{m} \sum_{\lambda_{1} \lambda_{2} \lambda_{3} \lambda_{4}} x^{(3)}\left(\lambda_{1} ; \lambda_{3}, \lambda_{2}, \lambda_{1}\right) \mathscr{G}_{\lambda_{1}} * \mathscr{G}_{\lambda_{3}} \mathscr{E}_{\lambda_{2}} * \mathscr{G}_{\lambda_{1}}
$$

